\title{
Arzneimittel- und Wirkstoffherstellungsverordnung (AMWHV): Wieviel Arzneimittelrecht braucht die Transfusionsmedizin noch?
}

\author{
Thomas Müller Ulrich Pohl \\ DRK Blutspendedienst NSTOB, Springe, Deutschland
}

\author{
Schlüsselwörter \\ Blutkomponenten - Synthetische Medikamente . \\ Arzneimittelgesetz · Transfusionsgesetz
}

\section{Zusammenfassung}

In Deutschland unterliegen Blutkomponenten zur Transfusion dem Arzneimittelgesetz (AMG) und damit auch den an das AMG gekoppelten Verordnungen wie der AMWHV. Die Herstellung und Prüfung von Blutkomponenten unterscheidet sich in wesentlichen Merkmalen von der konventioneller Arzneimittel. Sie umfasst insbesondere auch ärztliche Entscheidungen, die für synthetische Arzneimittel keine Bedeutung haben. Entsprechend führt die Einbeziehung von Blutkomponenten in das Arzneimittelrecht zu zahlreichen Sonderregelungen, die die Gewinnung konventioneller Arzneimittel ergänzen oder modifizieren. In der AMWHV als der Nachfolgerin der Pharmabetriebsverordnung ist den Blutkomponenten mit dem $\S 31$ erstmals ein eigener Abschnitt gewidmet. Im aktuellen AMG führen die speziellen Regelungen für Blutkomponenten zu einer erheblichen Komplexität, die der vernünftigen politischen Forderung nach einem überschaubaren Transfusionsrecht eindeutig widerspricht. Mit jeder Fortschreibung des Arzneimittelrechts in der EG und in Deutschland ist zu erwarten, dass die Zahl der speziellen Ausnahmeregelungen für Blutkomponenten weiter zunehmen wird. Deshalb bietet es sich an, die spezielle Regelung in Deutschland und in Österreich zu hinterfragen, die die Blutkomponenten im Unterschied zu allen anderen EG-Staaten dem Arzneimittelrecht unterwirft. Das Transfusionsgesetz eröffnet eine optimale rechtliche Grundlage, die EG-weit abgestimmten Anforderungen an die Gewinnung, Prüfung, Lagerung und den Vertrieb von Blutkomponenten außerhalb des AMG in überschaubarer Weise zu verankern.

\author{
Key Words \\ Blood components - Synthetic drugs - German Law on \\ Pharmaceuticals · German Law on Transfusion
}

\section{Summary}

The German Ordinance for the Production of Medicinal Products and Active Substances (AMWHV): Does the Assignment of Pharmaceutical Law to Blood Components in Germany Support Transfusion Medicine?

The preparation of blood components in Germany is regulated by the German Law on Pharmaceuticals (Arzneimittelgesetz; AMG) and thus by the German Ordinance for the Production of Medicinal Products and Active Substances (AMWHV). The preparation and quality assurance of blood components does, however, differ substantially from that of synthetic drugs. It especially includes medical decisions. The assignment of blood components to the AMG implicates numerous clauses confined to blood components or modifications of the rules for conventional drugs. The most recent version of the AMWHV reflects this status of blood components by introducing a new paragraph (§ 31) limited to blood components. The AMG is highly complicated due to the large number of clauses for blood components. It contradicts the political claim to keep the legal complexity in reasonable limits. Moreover, it is to be expected that future developments of the legal framework for pharmaceuticals will further increase the fundamental discrepancies in the requirements for synthetic drugs versus blood components. These considerations question the future benefits of the German and Austrian strategy to legally assign blood components to pharmaceuticals. The Council of Europe has clearly separated the legal framework for blood components from that for pharmaceuticals. The German Law on Transfusion does also offer an adequate tool to implement the European recommendations on the preparation, use and quality assurance of blood components.

\begin{tabular}{ll}
\hline KARGER & @ 2007 S. Karger GmbH, Freiburg \\
Fax +49761 4520714 & Accessible online at: \\
$\begin{array}{l}\text { E-mail Information@Karger.de } \\
\text { www.karger.com }\end{array}$ & www.karger.com/tmh
\end{tabular}




\section{Einleitung und Fragestellungen}

Nach dem Recht der Bundesrepublik Deutschland sind alle Blutzubereitungen, und damit auch alle humanen Blutkomponenten zur Transfusion, Arzneimittel ( $\$ 4$ Abs. 2 Arzneimittelgesetz, AMG) [1]. Unter Blutkomponenten (zur Transfusion) werden Frischplasmazubereitungen und Zubereitungen aus Blutzellen verstanden (Hämotherapie-Richtlinien 2005, Abschnitt 5) [2]. Die Definition als Arzneimittel bedeutet, dass die Gewinnung, die Lagerung und der Vertrieb dieser Produkte dem Arzneimittelgesetz, dem Heilmittelwerbegesetz und den europäischen Vorgaben der guten Herstellungspraxis (Good Manufacturing Practice; GMP) [3] unterliegen, sofern sie an andere abgegeben werden. Diese GMP-Vorgaben sind in Deutschland mit der Betriebsverordnung für pharmazeutische Unternehmer (PharmBetrV) in nationales Recht umgesetzt worden [4]. Die erste Fassung der PharmBetrV stammt vom 8. März 1985. In den vergangenen 20 Jahren wurde die PharmBetrV zehnmal geändert oder zumindest ergänzt. Diese Notwendigkeit regelmäßiger Modifikationen der PharmBetrV im 2-Jahres-Turnus verdeutlicht, dass die Anforderungen an pharmazeutische Unternehmen und damit auch an die Einrichtungen, die homologe Blutkomponenten herstellen, prüfen und in den Verkehr bringen, sich permanent weiterentwickelt haben. Die PharmBetrV in der Fassung vom 10. August 2004 [5] bzw. vom 10. Februar 2005 [6] führte mit ihren 20 Paragraphen in überschaubarer Struktur die allgemeinen Anforderungen an alle wesentlichen Elemente der Arzneimittelproduktion auf. Dazu gehörten die Ansprüche an das Qualitätssicherungssystem, das Personal, die Betriebsräume, die Hygiene, die Herstellung, die Prüfung (beides auch im Auftrag) und die Freigabe. Darüber hinaus wurden die Anforderungen an die Lagerung, die Tierhaltung, die Verpackung und Kennzeichnung, den Vertrieb und die Einfuhr, die Beanstandungen, die Dokumentation sowie die Selbstinspektion beschrieben. Nicht zuletzt wurde auch in $\S 17$ der PharmBetrV festgelegt, welche Verstöße gegen die Vorschriften der PharmBetrV als Ordnungswidrigkeiten gelten.

Seit dem 10. November 2006 ersetzt die AMWHV vom 3. November 2006 [7] die PharmBetrV. Ein wesentlicher Grund für die Ablösung liegt darin, dass EG-Vorgaben in Form von EGRichtlinien innerhalb bestimmter Fristen in nationales Recht $\mathrm{zu}$ übernehmen sind. So wurden mit der AMWHV 10 EGRichtlinien aus den Bereichen Human- und Tierarzneimittel, Blut und Blutbestandteile und menschliche Gewebe und Zellen in deutsches Recht umgesetzt. Die Erstellung der AMWHV gestaltete sich relativ schwierig, da auch über die EG-Vorgaben hinausgehende nationale Regelungen eingebracht werden sollten und sich die ursprünglich vorgesehene Strukturierung als zu komplex erwies. Die Vertreter der Bundesländer im Bundesrat koppelten ihre Zustimmung zum Entwurf der AMWHV des Bundesministeriums für Gesundheit und des Bundesministeriums für Ernährung, Landwirt- schaft und Verbraucherschutz an die Berücksichtigung zahlreicher Änderungswünsche [8].

Diese aktuellen, sehr umfassenden und zum Teil grundlegenden Veränderungen geben Anlass, die Auswirkungen der AMWHV auf die Transfusionsmedizin zu hinterfragen. Sie eröffnen die Chance, anhand des Vergleichs der Realitäten und der wünschenswerten Zukunft die rechtlichen Rahmenbedingungen in Deutschland für die Gewinnung von Blutkomponenten zu überdenken. Diese Überlegungen sollen unter Berücksichtigung des ärztlichen Selbstverständnisses helfen, rechtzeitig die Weichen für die Zukunft einer attraktiven und leistungsfähigen Transfusionsmedizin in Deutschland zu stellen.

\section{Die AMWHV}

Die AMWHV beinhaltet im Prinzip Regelungen für dieselben Bereiche der Arzneimittelproduktion wie die vorherige PharmBetrV. Sie erfasst aber auch zusätzlich die Arzneimittelwirkstoffherstellung. Die Struktur ist durch Gliederung in sieben Abschnitte, die vier verschiedene Regelungsbereiche umfassen, stark verändert und die Regelungstiefe - und damit der Umfang der Verordnung - mit jetzt 33 Paragraphen deutlich größer geworden. Die einzelnen Bereiche umfassen die allgemeinen Anforderungen, die Anforderungen an Arzneimittel, Blutprodukte und andere Blutbestandteile sowie Produkte menschlicher Herkunft, die Anforderungen für Wirkstoffe nichtmenschlicher Herkunft und die Sondervorschriften für Fütterungsarzneimittel und Blutspendeeinrichtungen. Die AMWHV ist jedoch nicht eigenständig, sondern wird erst ein vollständiges Regelwerk durch den EG-GMP-Leitfaden, auf den an vielen Stellen der Verordnung direkt verwiesen wird. Er ist daher jetzt auch in amtlicher deutscher Übersetzung verfügbar [9].

Blutspendeeinrichtungen und ihren besonderen Gegebenheiten wird im $\S 31$ der AMWHV Rechnung getragen. Dort sind die spezifischen Anforderungen an Blutspendeeinrichtungen, die deutlich von denen an andere Arzneimittelhersteller abweichen und letztlich auf der grundlegenden Blutrichtlinie 2002/98/EG [10] beruhen, ausführlich beschrieben:

- Das geforderte Qualitätsmanagementsystem muss die Anforderungen der technischen Blutrichtlinie 2005/62/EG [11] erfüllen, d.h., es muss eine speziell mit der Qualitätssicherung beauftragte Person vorhanden sein.

- Es müssen Arbeitsplatzbeschreibungen für das gesamte Personal, nicht nur für das in leitender oder für die GMPEinhaltung verantwortlicher Stellung, vorliegen.

- Die Besonderheiten der Betriebsräume, insbesondere bei mobilen Standorten, sind zu berücksichtigen.

- Die Herstellanweisung muss bezüglich der Spender die Erfüllung der Anforderungen des Anhangs II der Blutrichtlinie 2004/33/EG [12] ermöglichen.

- Die Prüfanweisung muss den Besonderheiten der Blutspende Rechnung tragen. 
- Es gibt die Möglichkeit einer Vertretungsregelung für die sachkundige Person bei der Freigabe von Blutzubereitungen für die unmittelbare Anwendung beim Menschen durch eine beauftragte Person.

\section{Blutspendedienste als "Arzneimittelhersteller", d.h. pharmazeutische Unternehmen mit speziellen Anforderungen}

Die AMWHV fasst im Unterschied zu der PharmBetrV als ihrer Vorgängerin im Abschnitt «Sonderregelungen» die besonderen Anforderungen an Blutspendeeinrichtungen in einem umfangreichen eigenen $\S 31$ zusammen. Damit wird den vielfältigen Alleinstellungsmerkmalen der Blutspendedienste, die sich als Hersteller und pharmazeutische Unternehmen auf Blutkomponenten beschränken, Rechnung getragen.

Für Transfusionsmediziner ist es selbstverständlich und klingt es nahezu trivial, dass sich die Vorgehensweisen und kritischen Determinanten zur Gewinnung von Blutzubereitungen, insbesondere Blutkomponenten, von den pharmazeutischen Prozessen zur Produktion konventioneller chemischer Arzneimittel in grundsätzlichen Merkmalen unterscheiden. Konventionelle Pharmaka werden heute, bis auf wenige Ausnahmen aus den Bereichen biologische, bio- und gentechnologische Arzneimittel, aus chemisch definierten Ausgangsstoffen zumindest im Kilo-, wenn nicht gar im Tonnenmaßstab synthetisiert. Die chemische Zusammensetzung einer Herstellcharge, die typischerweise mehrere Tausend bis hin zu Millionen Behandlungseinheiten umfasst, kann präzise analytisch charakterisiert werden. Damit kann die Person, die für die Qualitätskontrolle verantwortlich ist, anhand der Ergebnisse chemischer Analysen der Ausgangsstoffe, des chemisch definierten Wirkstoffs und der Hilfsstoffe für die Zubereitung des Arzneimittels die Qualität des fertigen Arzneimittels zuverlässig beurteilen. Auf dieser Basis gibt die sachkundige Person (die meist in Personalunion die Qualitätskontrolle leitet) mit einer Entscheidung eine Charge, und damit typischerweise eine große Zahl von Therapieeinheiten, frei.

Die Gewinnung von Blut und Blutbestandteilen beginnt, anders als bei allen anderen oben angeführten Arzneimitteln, mit der ärztlichen Anamnese - unterstützt vom und dokumentiert mit dem Spendebogen und der Untersuchung des Gesundheitszustands. Auf der Basis dieser ärztlichen Entscheidung wird die spendewillige Person zur Spende zugelassen. Aus der Spende wird ein kleines Volumen zum Nachweis biologischer Marker von Infektionen abgezweigt. Die Analyse der komplexen biologischen Qualitäten der gewonnenen Produkte mit ihren Myriaden unterschiedlicher Bestandteile bleibt jedoch im Vergleich zur Qualitätskontrolle konventioneller Arzneimittel sehr limitiert. Jedes Produkt aus der Spende eines Individuums stellt zumindest bei den meisten zellulären Produkten (Ausnahme: gepoolte Thrombozytenkonzen- trate) als therapeutische Einheit im Rahmen einer Einzelkomponentenherstellung eine eigene Charge dar, deren Rückverfolgung zum jeweiligen Spender gewährleistet werden muss. Der labormedizinische Nachweis von Infektionsmarkern im Blut des Spenders unter der Verantwortung der Leitung der Qualitätskontrolle stellt in diesem Kontext nur eines von mehreren unabhängigen Elementen zur optimalen Sicherheit der Blutprodukte dar. Die ärztliche Beurteilung der Spendefähigkeit vor der Entnahme sichert die Qualität und Sicherheit der Produkte im Rahmen der Aktivitäten, die die Leitung der Herstellung verantwortet. Probleme in diesem kritischen Bereich werden bei Blutzubereitungen im Unterschied zu den synthetischen konventionellen Pharmaka üblicherweise nicht bei In-Prozess-Kontrollen oder der vorgeschriebenen Prüfung des fertigen Arzneimittels erkannt. Damit erklärt sich ein wesentlich höherer Stellenwert für die unabhängige Verantwortung der Leitungen der Herstellung und der Qualitätskontrolle. Sie ist ein wesentlicher Garant der hohen Qualität und Sicherheit bei der Gewinnung von Blut und Blutbestandteilen.

In Anbetracht dieser fundamentalen Unterschiede überrascht es kaum, dass z.B. in der letzten Fassung der PharmBetrV die sachkundige Person zur Freigabe einer Arzneimittelcharge nicht von einer beauftragten Person vertreten werden durfte. Dies bedeutete, dass ein Erythrozytenkonzentrat, das beispielsweise notfallmäßig in der Nacht für einen immunsupprimierten Patienten bestrahlt werden musste, ebenfalls von der sachkundigen Person oder einer Vertretung gleicher Qualifikation vor dem Einsatz am Patienten kurzfristig freigegeben werden musste. Entsprechend hatte der Blutspendedienst als Arzneimittelhersteller sicherzustellen, dass für die kurzfristige Freigabe der Sonderpräparate unter den Blutkomponenten sachkundige Personen für $24 \mathrm{~h}$ am Tag 7 Tage in der Woche zur Verfügung standen. Eine Situation, die sich für konventionelle Arzneimittel nicht ergeben dürfte. Wie bereits erwähnt hat die AMWHV inzwischen mit der Möglichkeit nach $\S 31$, Beauftragte der sachkundigen Person ein zur unmittelbaren Anwendung am Menschen benötigtes Produkt vorläufig freigeben zu lassen, die Erfordernisse für diese spezielle Situation berücksichtigt.

Ein weniger kritisches, aber recht ärgerliches formales Problem bei der Anwendung des AMG auf Blutkomponenten ist die Verpackung. Bei konventionellen Arzneimitteln ist das Beifügen der Packungsbeilage mit den Anwendungs- und Warnhinweisen für die Patienten unbestrittenermaßen notwendig und sinnvoll. Bei Blutkomponenten werden die nach dem AMG erforderlichen Packungsbeilagen von den Anwendern in den Kliniken jedoch übereinstimmend als überflüssig und störend empfunden. Sie können sogar qualitätsmindernd sein, wenn sie - mehr oder weniger fest mit dem Blutbeutel verbunden - den notwendigen Gasaustausch behindern.

Sonderregelungen für Spezifikationen von Blutkomponenten finden sich auch im Abschnitt 3 der Hämotherapie-Richtlinien [2]: Zum einen werden regelmäßige Qualitätskontrollen 
an Stichproben hergestellter Blutkomponenten gefordert, d.h. dass nicht repräsentative Stichproben aus einer Charge, sondern ganze «Chargen» als Stichproben untersucht werden, da dies bei Blutkomponenten naturgemäß nicht anders möglich ist. Zum anderen wird die Einhaltung der jeweils angegebenen Spezifikation nur für $90 \%$ der geprüften Präparate gefordert. Beides sind Anforderungen, die von denen an konventionelle Arzneimittel stark abweichen.

\section{Rechtliche Randbedingungen für Blutspendedienste: «Keep it simple»}

Einen ersten Eindruck von der Komplexität, die das AMG der Bundesrepublik Deutschland [1] durch die Integration der unterschiedlichen Anforderungen an Produkte wie konventionelle synthetische Arzneimittel und Blutkomponenten abbilden muss, vermitteln die $\S \S 14$ und 15. Dort wird deutlich, dass die für die Herstellung verschiedener Blutzubereitungen zu erfüllenden Anforderungen grundsätzlich von denen für konventionelle Arzneimittel abweichen: In $§ 14$ sind die Qualifikationen der ärztlichen Personen aufgeführt, die Voraussetzung zur Erteilung einer Herstellungserlaubnis für Blutkomponenten sind. Dies ist etwas, das aufgrund der völlig anderen Gegebenheiten nicht im mindesten für die Herstellung von konventionellen Arzneimitteln gefordert wird. In $\S 15$ zählt der gesamte Absatz 3 die diversen Spezialanforderungen auf, die eine sachkundige Person für Blutzubereitungen erfüllen muss. Auch sie weichen von dem für konventionelle Arzneimittel Geforderten grundsätzlich ab.

Die zahlreichen Sonderregelungen für Blutzubereitungen sind im AMG nicht in einem einzigen Abschnitt zusammengefasst, sondern verstreut in den Gesamttext eingewoben. Damit ist die ursprüngliche, vor dem parlamentarischen Untersuchungsausschuss «HIV-Infektionen durch Blut und Blutprodukte» verbalisierte Forderung des ehemaligen Bundesgesundheitsministers Horst Seehofer nach Überschaubarkeit leider nicht mehr realisiert. Minister Seehofer forderte 1994: «Ich bin überzeugt, dass wir in Deutschland ... ein Transfusionsgesetz brauchen mit all diesen Sicherheitsnetzen, ... ein sauberes, überschaubares, in sich geschlossenes Recht, das Blut und Blutprodukte umfasst» [13].

Bietet es sich somit nach der Verabschiedung des Transfusionsgesetzes (TFG) in Deutschland nicht an, auf die ursprünglich sinnvolle Einbindung auch der Blutkomponenten in das deutsche AMG langfristig zu verzichten?

Dies entspräche zudem der allgemeinen Vorgehensweise in der EG. Außer in Deutschland sind Blutkomponenten nur noch in Österreich im Arzneimittelrecht verankert. Nicht nur die EG selbst, sondern auch alle anderen Mitgliedsstaaten haben für Blutkomponenten eigenständige Regelungen in Form von Richtlinien, Verordnungen oder Gesetzen unabhängig vom Arzneimittelrecht erlassen. Auch in der aktuellen Fassung der Richtlinie 2001/83/EG zur Schaffung eines
Gemeinschaftskodexes für Humanarzneimittel [14] ist im Artikel 3 ausdrücklich festgelegt, dass diese Richtlinie nicht gilt für «6. Vollblut, Plasma und Blutzellen menschlichen Ursprungs, mit Ausnahme des Plasmas, bei dessen Herstellung ein industrielles Verfahren zur Anwendung kommt». Dagegen existieren diverse EG-Blutrichtlinien, die sich spezifisch mit den Aspekten von Blut und Blutkomponenten befassen [10-12, 15]. Da sich das EG-Arzneimittelrecht konsequent weiter entwickeln wird, sollte die Abkoppelung der rechtlichen Grundlagen für Blutkomponenten in Deutschland vom nationalen Arzneimittelrecht, die dem EG-Recht definitiv nicht entgegen steht, unter anderem folgende Vorteile bieten:

- inhaltliche Fokussierung auf die Anforderungen, die speziell für Blutkomponenten relevant sind,

- auf Veränderungen in den Anforderungen an die Blutkomponenten reduzierter Aktualisierungsaufwand,

- Minimierung potenzieller Risiken und Missverständnisse durch bessere Überschaubarkeit der gesetzlichen Anforderungen, die von Nichtjuristen verstanden und umgesetzt werden müssen.

Diese Systematik unterstreicht auch die seit vielen Jahren bewährte Zuständigkeit des Paul-Ehrlich-Instituts als Bundesoberbehörde für Blutzubereitungen (und andere biologische Arzneimittel). Für die konventionellen Arzneimittel, die nicht aus Blut gewonnen werden, ist hingegen das Bundesinstitut für Arzneimittel und Medizinprodukte (BfArM) zuständig.

Jeder Zulassungsinhaber muss den Bundesoberbehörden regelmäßige aktualisierte Berichte über die Unbedenklichkeit des Arzneimittels vorlegen, die nicht arzneimittel-, sondern wirkstoffbezogen sein müssen. Bei Blutkomponenten ist eine derartige Vorgehensweise nicht zielführend, da eben nicht der Wirkstoff, sondern nur die Daten für die jeweilige Blutkomponente selbst, unter Berücksichtigung der Spenderauswahl und -testung sowie der spezifischen Herstellung, eine Beurteilung der Unbedenklichkeit erlauben.

Auch heute schon gibt es Unterschiede in den formalen Anforderungen an Zulassungsanträge beim Paul-Ehrlich-Institut für Blutkomponenten einerseits und andere Arzneimittel inklusive Plasmazubereitungen andererseits. Für Blutkomponenten gibt es ein vereinfachtes Verfahren [16, 17], während für andere Arzneimittel in der Zuständigkeit des Paul-Ehrlich-Instituts die üblichen Anforderungen nach den Vorgaben des «Common technical document»-Verfahrens (CTD-Verfahren) [18] einzuhalten sind.

Schon jetzt ist absehbar, dass es zum weiteren Auseinanderdriften von arzneimittelbezogenen Regelungen und Regelungen für Blutkomponenten kommen wird. So existiert bereits eine Regelung, die nicht sinnvoll auf Blutkomponenten anwendbar ist, in Form der EG-Kinderarzneimittel-Verordnung (EG) Nr. 1901/2006 vom 12.Dezember 2006 [19], die seit dem 26. Januar 2007 in Kraft ist und für Arzneimittel die Anforderungen zur Untersuchung von pädiatrischen Indikationen oder zur Freistellung davon regelt. 
Bereits getrennte Wege für Arzneimittel und Blut- und Gewebezubereitungen zeigt der aktuelle Gewebegesetzentwurf der Bundesregierung [20] auf, wenn auch beide Wege im AMG enthalten sein sollen. Vorgesehen ist, die Meldeverpflichtungen für Arzneimittel-Nebenwirkungsverdachtsfälle mit dem zukünftig veränderten $§ 63 \mathrm{~b}$ AMG und die Meldeverpflichtungen von schwerwiegenden unerwünschten Zwischenfällen und Reaktionen bei Blut- und Gewebezubereitungen in Umsetzung der entsprechenden EG-Richtlinie mit dem neuen $\S 63$ c AMG deutlich unterschiedlich zu regeln [15].

Falls der Automatismus der Übernahme der arzneimittelbezogenen EG-Regelungen auch für Blutkomponenten in Deutschland weitergeführt wird, werden in Zukunft noch weitere Probleme entstehen.

\section{Schlussfolgerungen}

Die gesetzlichen Anforderungen an Arzneimittel werden sich auch in Zukunft stringent weiterentwickeln. Entsprechend ist zu erwarten, dass sich die speziellen Erfordernisse für die Herstellung von Blutkomponenten, die nicht allein pharmazeutischen Ansprüchen genügen müssen, sondern auch ganz wesentlich ärztliche Entscheidungen beinhalten, weiter differenzieren.

Vor dem Hintergrund der zukünftigen Herausforderungen erscheint es sinnvoll, die in Deutschland etablierte Definition von Blutzubereitungen als Arzneimittel abzulösen. In Deutschland wäre mit der ausschließlichen Verankerung der Blutkomponenten im TFG $[6,21]$ auch bei Beendigung der Klassifikation als Arzneimittel der EG-weit akzeptierte Sonderstatus von Blutkomponenten relativ einfach zu realisieren. Dieser fundamentale Schritt könnte die Komplexität der gesetzlichen Anforderungen an die Transfusionsmedizin in Deutschland (und gegebenenfalls in Österreich) erheblich reduzieren. Gleichzeitig hilft er, den in einer Zeit des Ärztemangels bedeutsamen Verlust der Attraktivität der Weiterbildung in Transfusionsmedizin zu kompensieren. So sollte es gelingen, das Fachgebiet des Transfusionsmediziners langfristig von überflüssigem Ballast zu befreien.

\section{Literatur}

1 Vierzehntes Gesetz zur Änderung des Arzneimittelgesetzes vom 29. August 2005. Bundesgesetzblatt I S. 2570 .

2 Richtlinien zur Gewinnung von Blut und Blutbestandteilen und zur Anwendung von Blutprodukten (Hämotherapie), aufgestellt von der Bundesärztekammer im Einvernehmen mit dem Paul-Ehrlich-Institut (Novelle 2005). Bundesanzeiger Nr. 209a, 5.11.2005.

3 EUDRALEX Volume 4 - Medicinal Products for Human and Veterinary Use: Good Manufacturing Practice http://ec.europa/enterprise/pharmaceuticals/ eudralex/homev4.htm.

4 Betriebsverordnung für pharmazeutische Unternehmer vom 8. März 1985. Bundesgesetzblatt I S.546.

5 Dritte Verordnung zur Änderung der Betriebsverordnung für pharmazeutische Unternehmer vom 10. August 2004. Bundesgesetzblatt I, S.2155.

6 Erstes Gesetz zur Änderung des Transfusionsgesetzes und arzneimittelrechtlicher Vorschriften vom 10. Februar 2005. Bundesgesetzblatt I, S.234.

7 Verordnung zur Ablösung der Betriebsverordnung für pharmazeutische Unternehmer vom 3. November 2006. Bundesgesetzblatt I, S.2523.

8 Bundesratsdrucksache 398/06 (Beschluss) vom 7.7.2006 zur Verordnung: zur Ablösung der Betriebsverordnung für pharmazeutische Unternehmer. www.bundesrat.de.

9 Bekanntmachung zu $\$ 2$ Nr. 3 der Arzneimittelund Wirkstoffherstellungsverordnung vom 26.10. 2006, Anlage 1: Eudralex - Die Regelung der Arzneimittel in der Europäischen Union, Band 4, EU Leitlinien für die Gute Herstellungspraxis Humanarzneimittel und Tierarzneimittel. Bundesanzeiger Nr. 210, 09.11.2006, S. 6887.
10 Richtlinie 2002/98/EG des Europäischen Parlaments und des Rates vom 27. Januar 2003 zur Festlegung von Qualitäts- und Sicherheitsstandards für die Gewinnung, Testung, Verarbeitung, Lagerung und Verteilung von menschlichem Blut und Blutbestandteilen und zur Änderung der Richtlinie 2001/83/EG. Amtsblatt der Europäischen Union L33/30-40.

11 Richtlinie 2005/62/EG der Kommission vom 30. September 2005 zur Durchführung der Richtlinie 2002/98/EG des Europäischen Parlaments und des Rates in Bezug auf gemeinschaftliche Standards und Spezifikationen für ein Qualitätssystem für Blutspendeeinrichtungen. Amtsblatt der Europäischen Union L 256/41-46.

12 Richtlinie 2004/33/EG der Kommission vom 22. März 2004 zur Durchführung der Richtlinie 2002/ 98/EG des Europäischen Parlaments und des Rates hinsichtlich bestimmter technischer Anforderungen für Blut und Blutbestandteile. Amtsblatt der Europäischen Union L91/25-39.

13 Zitat: Minister Seehofer in 1994, wiedergegeben in der Bundestagdrucksache 12/8591, S. 255; in Deutsch E, Bender AW, Eckstein R, Zimmermann R (Hrg): Transfusionsrecht. Stuttgart, Wissenschaftliche Verlagsgesellschaft, 2001, S. 13.

14 Richtlinie 2004/27/EG des Europäischen Parlaments und des Rates vom 31. März 2004 zur Änderung der Richtlinie 2001/83/EG zur Schaffung eines Gemeinschaftskodexes für Humanarzneimittel. Amtsblatt der Europäischen Union L 136/34-57.

15 Richtlinie 2005/61/EG der Kommission vom 30. September 2005 zur Durchführung der Richtlinie 2002/98/EG des Europäischen Parlaments und des Rates in Bezug auf die Anforderungen an die Rückverfolgbarkeit und die Meldung ernster Zwischenfälle und ernster unerwünschter Reaktionen. Amtsblatt der Europäischen Union L256/ 32-40.
16 Bekanntmachung über die Zulassung von Arzneimitteln (Neuer Formularsatz für Anträge auf $\mathrm{Zu}$ lassung von Blutkomponenten zur Transfusion) vom 26.11.2003. Bundesanzeiger Nr. 15a, 23.01. 2004, S. 1.

17 Unterlagen zur Zulassung von Blutkomponenten zur Transfusion, www.pei.de/cln_049/nn_157160/ DE/infos/pu/03-zul-hum/06-blut/antrag-zulassung.html.

18 Bekanntmachung über die Zulassung von Arzneimitteln - Neuer Formularsatz für den Antrag auf Zulassung eines Arzneimittels beim Bundesam für Arzneimittel und Medizinprodukte und beim Paul-Ehrlich-Institut/Bundesamt für Sera und Impfstoffe vom 21.01.2003. Bundesanzeiger Nr. 69a, 09.04.2003, S. 1.

19 Verordnung (EG) Nr. 1901/2006 des Europäischen Parlaments und des Rates vom 12. Dezember 2006 über Kinderarzneimittel und zur Änderung der Verordnung (EWG) Nr. 1768/92, der Richtlinien 2001/20/EG und 2001/83/EG sowie der Verordnung (EG) Nr. 726/2004. Amtsblatt der Europäischen Union L 378/1-19.

20 Gesetzentwurf der Bundesregierung: Entwurf eines Gesetzes über Qualität und Sicherheit von menschlichen Geweben und Zellen (Gewebegesetz). Bundestagsdrucksache 16/3146. www.bundestag.de/ ausschuesse/a14/anhoerungen/044/BT-Drs 2006.

21 Gesetz zur Regelung des Transfusionswesens (Transfusionsgesetz - TFG) vom 1. Juli 1998 Bundesgesetzblatt I S.1752. 